\title{
Designing Commutative Cascades of Multidimensional Upsamplers and Downsamplers
}

Brian L. Evans, Member, IEEE

\begin{abstract}
In multiple dimensions, the cascade of an upsampler by $\mathbf{L}$ and a downsampler by $\mathbf{L}$ commutes if and only if the integer matrices $\mathbf{L}$ and $\mathbf{M}$ are right coprime and $\mathbf{L M}=$ ML. This paper presents algorithms to design $\mathbf{L}$ and $\mathbf{M}$ that yield commutative upsampler/dowsampler cascades. We prove that commutativity is possible if the Jordan canonical form of the rational (resampling) matrix $\mathbf{R}=\mathbf{L} \mathbf{M}^{-1}$ is equivalent to the Smith-McMillan form of $\mathbf{R}$. A necessary condition for this equivalence is that $\mathbf{R}$ has an eigendecomposition and the eigenvalues are rational.
\end{abstract}

B. L. Evans is with the Department of Electrical and Computer Engineering, The University of Texas at Austin, Austin, TX 78712-1084, USA. E-mail: bevans@ece.utexas.edu, Web: http://www.ece.utexas.edu/ ${ }^{\sim}$ bevans, Phone: (512) 232-1457, Fax: (512) 471-5907.

This work was sponsored in part by NSF CAREER Award under Grant MIP-9702707. 


\section{INTRODUCTION}

Resampling systems scale the sampling rate by a rational factor $R=L / M=L M^{-1}$, or equivalently decimate by $H=M / L=L^{-1} M$ [1], by essentially upsampling by $L$, filtering, and downsampling by $M$. In converting compact disc data sampled at $44.1 \mathrm{kHz}$ to digital audio tape data sampled at $48 \mathrm{kHz}, R=\frac{48000 \mathrm{~Hz}}{44100 \mathrm{~Hz}}=\frac{160}{147}$. Because we can always factor $R$ into coprime integers $L$ and $M$, we can always commute the upsampler and downsampler which leads to efficient polyphase structures of the resampling system. In multiple dimensions, resampling is described by a rational matrix $\mathbf{R}$. Multidimensional resampling systems are essentially a cascade of an upsampler by $\mathbf{L}$, a filter, and a downsampler by $\mathbf{M}$, such that $\mathbf{R}=\mathbf{L ~ M}^{-1}$ and $\mathbf{L}$ and $\mathbf{M}$ are non-singular integer matrices. Although it is rare that a cascade of an upsampler and downsampler commutes in multiple dimensions, we can nonetheless always find polyphase structures for multidimensional resampling systems. Polyphase structures exist because we can always factor $\mathbf{R}$ into right coprime $\mathbf{L}$ and $\mathbf{M}$ which is known as relaxed commutativity [2].

Commutativity of a multidimensional upsampler and downsampler in cascade [2], [3], [4], [5] occurs when

1. $\quad \mathbf{L}$ and $\mathbf{M}$ are right coprime, and

\section{2. $\mathbf{L M}=\mathbf{M L}$}

Many approaches exist for checking whether or not a cascade commutes, given values of $\mathbf{L}$ and $\mathbf{M}$. Techniques exist for generating $\mathbf{L}$ and $\mathbf{M}$ that are right coprime by decomposing $\mathbf{R}$ into its Smith-McMillan form [6]. Finding algorithms to generate cascades that satisfy both commutativity conditions is an open problem [7]. These algorithms play a role in rearranging [4] and scheduling [8] multidimensional multirate systems.

In the paper, we develop two algorithms to design $\mathbf{L}$ and $\mathbf{M}$ that yield commutative cascades:

1. generate $\mathbf{L}$ and $\mathbf{M}$, and

2. factor a desired $\mathbf{R}$ into $\mathbf{L} \mathbf{M}^{-1}$

(if $\hat{\mathbf{L}}$ and $\hat{\mathbf{M}}$ are given, use $\mathbf{R}=\hat{\mathbf{L}} \hat{\mathbf{M}}^{-1}$ )

The key to the new algorithms is that we can satisfy both commutativity conditions. In particular, we can satisfy the condition that $\mathbf{L M}=\mathbf{M L}$ if the Jordan canonical form of $\mathbf{R}=\mathbf{L ~ M}^{-1}$ is equivalent to a Smith-McMillan form. We show that a necessary condition for this equivalence is that $\mathbf{R}$ has an eigendecomposition and the eigenvalues are rational. Once we satisfy $\mathbf{L M}=\mathbf{M L}$ using our approach, we automatically satisfy the other commutativity condition. 


\section{BACKGROUND}

In multiple dimensions, downsampling is described by a non-singular integer downsampling matrix M. On average, a downsampler outputs one sample for every group of $|\operatorname{det} \mathbf{M}|$ input samples. Its response $x_{d}[\mathbf{n}]$ to input $x[\mathbf{n}]$ is

$$
x_{d}[\mathbf{n}]=x[\mathbf{M n}]
$$

Upsampling is described by a non-singular integer upsampling matrix $\mathbf{L}$. It outputs $|\operatorname{det} \mathbf{L}|$ samples for every input sample. Its response $x_{\mathrm{u}}[\mathbf{n}]$ to input $x[\mathbf{n}]$ is

$$
x_{\mathrm{u}}[\mathbf{n}]=\left\{\begin{array}{cl}
x\left[\mathbf{L}^{-1} \mathbf{n}\right] & \text { if } \mathbf{L}^{-1} \mathbf{n} \in \mathcal{R}_{I} \\
0 & \text { otherwise }
\end{array}\right.
$$

An upsampler/downsampler cascade commutes if $\mathbf{L}$ and $\mathbf{M}$ meet the conditions in (1). The Smith form decomposition is useful for testing [4] and generating [6] $\mathbf{L}$ and $\mathbf{M}$ to meet the right coprime condition. The Smith form decomposition of a non-singular integer matrix $\mathbf{S}$ is

$$
\mathbf{S}=\mathbf{U}_{\mathbf{S}} \Lambda_{\mathbf{S}} \mathbf{V}_{\mathbf{S}}
$$

where $\boldsymbol{\Lambda}_{\mathbf{S}}$ is a diagonal integer matrix and $\mathbf{U}_{\mathbf{S}}$ and $\mathbf{V}_{\mathbf{S}}$ are unimodular integer matrices (a unimodular matrix has a determinant of \pm 1 ). The Smith form decomposition decouples a linear operation and is not unique. When applied to non-singular rational matrix, it is called the SmithMcMillan form decomposition, and has the same form as (4) but $\boldsymbol{\Lambda}$ is a diagonal rational matrix. The Smith form decomposition always exists for non-singular integer and rational matrices [9].

Given a rational resampling matrix $\mathbf{R}$, or given $\hat{\mathbf{L}}$ and $\hat{\mathbf{M}}$ and forming $\mathbf{R}=\hat{\mathbf{L}} \hat{\mathbf{M}}^{-1}$, we can always factor $\mathbf{R}=\mathbf{L} \mathbf{M}^{-1}$ such that $\mathbf{L}$ and $\mathbf{M}$ are right coprime using the Smith form decomposition of $\mathbf{R}[6]$ :

$$
\begin{aligned}
\mathbf{R} & =\mathbf{U} \boldsymbol{\Lambda} \mathbf{V} \\
& =\mathbf{U} \boldsymbol{\Lambda}_{\mathbf{L}} \boldsymbol{\Lambda}_{\mathbf{M}}^{-1} \mathbf{V} \\
& =\left(\mathbf{U} \boldsymbol{\Lambda}_{\mathbf{L}}\right)\left(\mathbf{V}^{-1} \boldsymbol{\Lambda}_{\mathbf{M}}\right)^{-1} \\
& =\mathbf{L} \mathbf{M}^{-1}
\end{aligned}
$$

$\boldsymbol{\Lambda}$ is a diagonal rational matrix, $\boldsymbol{\Lambda}=\boldsymbol{\Lambda}_{\mathbf{L}} \boldsymbol{\Lambda}_{\mathbf{M}}{ }^{-1}, \boldsymbol{\Lambda}_{\mathbf{L}}$ and $\boldsymbol{\Lambda}_{\mathbf{M}}$ are diagonal integer matrices, and

$$
\mathbf{L}=\mathbf{U} \boldsymbol{\Lambda}_{\mathbf{L}} \text { and } \mathbf{M}=\mathbf{V}^{-1} \boldsymbol{\Lambda}_{\mathbf{M}}
$$

Since $\mathbf{V}$ is unimodular, $\mathbf{V}^{-1}$ is unimodular. So, $\mathbf{L}$ and $\mathbf{M}$ are always right coprime provided that each rational number on the diagonal of $\boldsymbol{\Lambda}$ is reduced to $\frac{l}{m}$ where $l$ and $m$ are coprime integers. 


\section{iII. Designing Commutative Cascades}

In this section, we determine the conditions for which $\mathbf{L}$ and $\mathbf{M}$ satisfy the commutativity conditions given by (1). Since $\mathbf{L}$ and $\mathbf{M}$ are non-singular, the condition $\mathbf{L M}=\mathbf{M L}$ holds when $\mathbf{L}$ and $\mathbf{M}$ have the same eigenvector matrix denoted by $\mathbf{U}[10]$

$$
\mathbf{L}=\mathbf{U} \boldsymbol{\Lambda}_{\mathbf{L}} \mathbf{U}^{-1} \text { and } \mathbf{M}=\mathbf{U} \boldsymbol{\Lambda}_{\mathbf{M}} \mathbf{U}^{-1}
$$

because

$$
\begin{aligned}
\mathbf{L} \mathbf{M} & =\left(\mathbf{U} \boldsymbol{\Lambda}_{\mathbf{L}} \mathbf{U}^{-1}\right)\left(\mathbf{U} \boldsymbol{\Lambda}_{\mathbf{M}} \mathbf{U}^{-1}\right) \\
& =\mathbf{U} \boldsymbol{\Lambda}_{\mathbf{L}} \boldsymbol{\Lambda}_{\mathbf{M}} \mathbf{U}^{-1} \\
& =\mathbf{U} \boldsymbol{\Lambda}_{\mathbf{M}} \boldsymbol{\Lambda}_{\mathbf{L}} \mathbf{U}^{-1} \\
& =\mathbf{M} \mathbf{L}
\end{aligned}
$$

Notice that $\mathbf{L}$ and $\mathbf{M}$ are coprime when $\boldsymbol{\Lambda}_{\mathbf{L}}$ and $\boldsymbol{\Lambda}_{\mathbf{M}}$ are coprime. Forming the rational matrix

$$
\mathbf{R}=\mathbf{L} \mathbf{M}^{-1}=\mathbf{U} \boldsymbol{\Lambda} \mathbf{U}^{-1}
$$

we develop an algorithm to generate $\mathbf{L}$ and $\mathbf{M}$, and another to determine when and how to factor a given $\mathbf{R}$ matrix into $\mathbf{L}$ and $\mathbf{M}$, that satisfy the commutativity conditions.

Since a non-singular matrix does not always have an eigendecomposition, such as

$$
\left[\begin{array}{lll}
1 & 1 & 0 \\
0 & 1 & 1 \\
0 & 0 & 1
\end{array}\right]
$$

which cannot be diagonalized, we use the Jordan canonical form which always exists for a nonsingular matrix [10]. The Jordan canonical form decomposes a non-singular matrix $\mathbf{R}$ into $\mathbf{S ~ J ~ S} \mathbf{S}^{-1}$. When $\mathbf{J}$ is a diagonal matrix, the Jordan canonical form is an eigendecomposition of $\mathbf{R}$ in which the diagonal elements of $\mathbf{J}$ are the eigenvalues and the columns of $\mathbf{S}$ are the eigenvectors corresponding to the eigenvalues [10]. In our case, $\mathbf{R}$ is a rational (resampling) matrix.

Theorem 1 states that we can always find a rational eigenvector matrix for a rational matrix when the eigenvalues are rational. Theorem 2 defines equivalence between the eigendecomposition and Smith form decomposition. Theorem 3 determines how to compute (7) from $\mathbf{R}$ when Theorem 2 applies.

Theorem 1: Given a square non-singular rational matrix $\mathbf{R}$ with Jordan canonical form $\mathbf{R}=\hat{\mathbf{S}} \mathbf{J} \hat{\mathbf{S}}^{-1}$, if $\mathbf{J}$ is a diagonal rational matrix, then we can always find an alternative Jordan canonical form $\mathbf{R}=\mathbf{S} \mathbf{J ~ S}^{-1}$ in which $\mathbf{S}$ is a rational matrix. 
Proof: If $\mathbf{J}$ is diagonal, then the Jordan canonical form of $\mathbf{R}$ is an eigendecomposition of $\mathbf{R}$. Define $\hat{\mathbf{s}}_{i}$ to be the $i$ th column vector (eigenvector) of $\hat{\mathbf{S}}$. Therefore, $\hat{\mathbf{s}}_{i}$ satisfies

$$
\mathbf{R} \hat{\mathbf{s}}_{i}=\lambda_{i} \hat{\mathbf{s}}_{i}
$$

where $\lambda_{i}=\mathbf{J}_{i i}$, i.e., the $i$ th eigenvalue. An eigenvector is unique only up to a scale factor, so $\hat{\mathbf{s}}_{i}=\alpha_{i} \mathbf{s}_{i}$ where $\alpha$ is a scalar. Since $\mathbf{R}$ is a rational matrix and $\lambda_{i}$ is a rational number, we can always compute $\alpha$ by taking the gcd of the non-zero elements of $\hat{\mathbf{s}}_{i}$ to yield a rational eigenvector $\mathbf{s}_{i}=\frac{1}{\alpha} \hat{\mathbf{s}}_{i}$. Then, we construct $\mathbf{S}$ by setting its $i$ th column vector to be equal to $\mathbf{s}_{i}$ for all $i$. QED

Theorem 2: An eigendecomposition of a non-singular rational matrix $\mathbf{R}=\mathbf{S} \mathbf{J} \mathbf{S}^{-1}$ is equivalent to the Smith-McMillan decomposition of $\mathbf{R}=\mathbf{U} \mathbf{\Lambda} \mathbf{V}^{-1}$ if the eigenvalues of $\mathbf{R}$ given by the diagonal elements of $\mathbf{J}$ are rational and there exists a rational diagonal matrix $\mathbf{D}$ for which $\mathbf{U}=\mathbf{S} \mathbf{D}$ is a unimodular integer matrix.

Proof: Let $\mathbf{D}$ be a diagonal matrix. Generalize the eigendecomposition of $\mathbf{R}$ as

$$
\mathbf{R}=\mathbf{S} \mathbf{J} \mathbf{S}^{-1}=\mathbf{S} \mathbf{J} \mathbf{D} \mathbf{D}^{-1} \mathbf{S}^{-1}=(\mathbf{S} \mathbf{D}) \mathbf{J}(\mathbf{S} \mathbf{D})^{-1}
$$

since the product of diagonal matrices commutes $(\mathbf{J} \mathbf{D}=\mathbf{D} \mathbf{J})$. Equating the general eigendecomposition and the Smith-McMillan decomposition, we get

$$
\mathbf{J}=\boldsymbol{\Lambda} \text { and } \mathbf{U}=\mathbf{S} \mathbf{D}
$$

Since $\boldsymbol{\Lambda}$ is a diagonal rational matrix, $\mathbf{J}$ is a diagonal rational matrix, which means that $\mathbf{R}$ has rational eigenvalues. Because $\mathbf{S}$ is rational, $\mathbf{D}$ is a rational matrix to ensure that $\mathbf{U}$ is an integer matrix. We chose $\mathbf{D}$ with the smallest absolute determinant that makes $\mathbf{U}$ be an integer matrix. Let $\mathbf{s}_{i}$ be the $i$ th column vector of $\mathbf{S}$ and $\mathbf{z}_{i}$ be the set of the non-zero elements of $\mathbf{s}_{i}$. Let $\mathbf{D}_{i i}=d_{i}$ where $d_{i}$ is the ratio of the lcm of the denominators of $\mathbf{z}_{i}$ over the lcm of the numerators of $\mathbf{z}_{i}$. The product $d_{i} \mathbf{s}_{i}$ is the integer vector with smallest $L_{p}$ norm in the direction of the eigenvector $\mathbf{s}_{i}$. Since $\mathbf{U}$ is integer and non-singular, $|\operatorname{det} \mathbf{U}| \geq 1$. Because $\mathbf{S}$ is non-singular,

$$
|\operatorname{det} \mathbf{U}|=|\operatorname{det} \mathbf{S}||\operatorname{det} \mathbf{D}|=|\operatorname{det} \mathbf{S}| \prod_{i} d_{i}
$$

and any other $\mathbf{D}$ that guarantees that $\mathbf{U}$ is an integer matrix cannot decrease $|\operatorname{det} \mathbf{U}|$. Therefore, there is an equivalence between an eigendecomposition of a rational matrix and its SmithMcMillan form if the eigenvalues are rational and $\operatorname{det} \mathbf{U}= \pm 1$. QED 
Theorem 3: Given a square non-singular rational matrix $\mathbf{R}$ with an eigendecomposition, if the eigendecomposition of $\mathbf{R}$ is equivalent to the Smith-McMillan decomposition of $\mathbf{R}$, then we can compute the integer matrices $\mathbf{L}$ and $\mathbf{M}$ such that $\mathbf{R}=\mathbf{L ~ M}^{-1}, \mathbf{L} \mathbf{M}=\mathbf{M} \mathbf{L}$, and $\mathbf{L}$ and $\mathbf{M}$.

Proof: If the eigendecomposition and Smith-McMillan decomposition of $\mathbf{R}$ are equivalent, then

$$
\mathbf{R}=\mathbf{S} \mathbf{J} \mathbf{S}^{-1}=\mathbf{U} \boldsymbol{\Lambda} \mathbf{U}^{-1}
$$

from Theorem 2. Set $\mathbf{U}=\mathbf{S} \mathbf{D}$ and $\boldsymbol{\Lambda}=\mathbf{J}$. Use (7) to compute $\mathbf{L}$ and $\mathbf{M}$. QED

Figure 1 shows an algorithm based on the results of Theorems 1-3 to factor a rational resampling matrix $\mathbf{R}$ into $\mathbf{L}$ and $\mathbf{M}$ that satisfy the commutativity conditions given in (7). If instead we are given $\hat{\mathbf{L}}$ and $\hat{\mathbf{M}}$, we input $\mathbf{R}=\hat{\mathbf{L}} \hat{\mathbf{M}}^{-1}$ to the algorithm. Figure 2 applies the algorithm to three resampling matrices. The resampling matrices in Figure 2(a) and 2(b) have rational eigenvalues. Using their Jordan canonical form representations, (7) is used to compute $\mathbf{L}$ and $\mathbf{M}$. Therefore, $\mathbf{L}$ and $\mathbf{M}$ satisfy the commutativity conditions in (1). The resampling matrix in Figure 2(c) has irrational eigenvalues. Since the eigenvalues are not rational, the Smith Form Decomposition of the resampling matrix is used to compute $\mathbf{L}$ and $\mathbf{M}$ according to (6). Therefore, $\mathbf{L}$ and $\mathbf{M}$ are right coprime, but do not commute under matrix multiplication.

We now turn our attention to generating new rational resampling matrices of the form $\mathbf{R}=$ $\mathbf{U} \boldsymbol{\Lambda} \mathbf{U}^{-1}$. We use (8) to compute $\mathbf{L}$ and $\mathbf{M}$ that satisfy the commutativity conditions in (1). We have freedom to choose a non-singular diagonal rational matrix $\boldsymbol{\Lambda}$ and a non-singular integer matrix $\mathbf{U}$, which are both $m \times m$ matrices. $\mathbf{U}$ has $m^{2}$ integer elements but must be unimodular, and $\boldsymbol{\Lambda}$ has $m$ non-zero rational entries. So, for $m$ dimensions, we have the freedom to choose $m^{2}+2 m-1$ integers, e.g. 7 integers in two dimensions and 14 integers in three dimensions. The examples of rational resampling matrices in Figure 2(a) and 2(b) were generated in this manner.

\section{Conclusion}

We present two algorithms for designing commutative upsampler/downsampler cascades: one generates the cascades, and the other tries to find a commutative cascade that performs a desired resampling. Both algorithms are based on Theorem 2 that states that commutativity occurs when the Jordan canonical form of the rational resampling matrix is equivalent to the Smith-McMillan form of the rational resampling matrix. A necessary condition for this equivalence is that the rational resampling matrix has an eigendecomposition and the eigenvalues are rational. 


\section{REFERENCES}

[1] P. P. Vaidyanathan, Multirate Systems and Filter Banks. Englewood Cliffs, NJ: Prentice Hall, 1993.

[2] T. Chen and P. P. Vaidyanathan, "The role of integer matrices in multidimensional multirate systems," IEEE Trans. on Signal Processing, vol. 41, pp. 1035-1047, Mar. 1993.

[3] A. A. Kalker, "Commutativity of up/down sampling," IEE Electronics Letters, vol. 28, pp. 567-569, Mar. 1992.

[4] B. L. Evans, R. H. Bamberger, and J. H. McClellan, "Rules for multidimensional multirate structures," IEEE Trans. on Signal Processing, vol. 42, pp. 762-771, Apr. 1994.

[5] R. A. Gopinath and C. S. Burrus, "On upsampling, downsampling and rational sampling rate filter banks," IEEE Trans. on Signal Processing, vol. 42, pp. 812-824, Apr. 1995.

[6] B. L. Evans, A Knowledge-Based Environment for the Design and Analysis of Multidimensional Multirate Signal Processing Algorithms. PhD thesis, Georgia Institute of Technology, Atlanta, GA, June 1993. (Available by FTP to ftp.eedsp.gatech.edu as Mathematica/evans_thesis.ps.Z).

[7] M. R. Khansari and T. Chen, "Finding commutative multidimensional downsamplers and upsamplers," IEEE Trans. on Signal Processing, vol. 43, pp. 2002-2004, Aug. 1995.

[8] P. K. Murthy and E. A. Lee, "An extension of multidimensional synchronous dataflow to handle arbitrary sampling lattices," in Proc. IEEE Int. Conf. Acoust., Speech, and Signal Processing, vol. 6, (Atlanta, GA), pp. 3306-3309, May 1996.

[9] A. Kaufmann and A. Henry-Labordère, Integer and Mixed Programming: Theory and Applications. New York: Academic Press, 1977.

[10] G. Strang, Linear Algebra and Its Applications. New York: Academic Press, 1980.

[11] B. Char, K. Geddes, G. Gonnet, B. Leong, M. Monagan, and S. Watt, First Leaves: A Tutorial Introduction to Maple V. New York: Springer-Verlag, 1992.

[12] S. Wolfram, The Mathematica Book. Champaign, IL: Wolfram Media Inc., third ed., 1996. 
1. Input a square non-singular rational matrix $\mathbf{R}$ in exact precision.

2. Compute the Jordan canonical form $\mathbf{R}=\mathbf{S} \mathbf{J ~ S}^{-1}$ in exact precision, e.g., using Maple [11] or Mathematica [12]. †

3. If Theorem 2 applies for $\mathbf{R}$, then use the Jordan canonical form of $\mathbf{R}$ to compute $\mathbf{L}$ and $\mathbf{M}$ according to Theorem 3 .

4. Otherwise, compute the Smith form decomposition of $\mathbf{R}$, and use the Smith form decomposition to compute $\mathbf{L}$ and $\mathbf{M}$ according to (6).

$\dagger$ If $\mathbf{J}$ is a rational diagonal matrix and $\mathbf{S}$ is not a rational matrix, then apply Theorem 1 to recompute $\mathbf{R}=\mathbf{S} \mathbf{J} \mathbf{S}^{-1}$ so that $\mathbf{S}$ is a rational matrix.

Fig. 1. An algorithm based on the Jordan canonical form to try to decompose a rational matrix $\mathbf{R}$ into $\mathbf{R}=\mathbf{L} \mathbf{M}^{-1}$ to meet the two commutativity conditions in (1). 


$$
\begin{gathered}
{\left[\begin{array}{cc}
\frac{17}{3} & -\frac{5}{3} \\
\frac{25}{2} & -\frac{7}{2}
\end{array}\right]=\left[\begin{array}{ll}
\frac{1}{3} & \frac{2}{5} \\
1 & 1
\end{array}\right] \quad\left[\begin{array}{ll}
\frac{2}{3} & 0 \\
0 & \frac{3}{2}
\end{array}\right]\left[\begin{array}{cc}
-15 & 6 \\
15 & -5
\end{array}\right]} \\
\mathbf{L}=\left[\begin{array}{cc}
8 & -2 \\
15 & -3
\end{array}\right] \quad \mathbf{M}=\left[\begin{array}{ll}
-3 & 2 \\
-15 & 8
\end{array}\right]
\end{gathered}
$$

(a) A two-dimensional commutative cascade

$$
\begin{gathered}
{\left[\begin{array}{ccc}
\frac{3}{2} & -1 & \frac{1}{2} \\
4 & -2 & -1 \\
-5 & 2 & 4
\end{array}\right]=\left[\begin{array}{ccc}
\frac{3}{2} & 1 & \frac{1}{2} \\
2 & 1 & \frac{1}{4} \\
1 & 1 & 1
\end{array}\right]\left[\begin{array}{ccc}
\frac{1}{2} & 0 & 0 \\
0 & 1 & 0 \\
0 & 0 & 2
\end{array}\right]\left[\begin{array}{ccc}
-6 & 4 & 2 \\
14 & -8 & -5 \\
-8 & 4 & 4
\end{array}\right]} \\
\mathbf{L}=\left[\begin{array}{ccc}
-3 & 2 & 2 \\
-2 & 2 & 1 \\
-8 & 4 & 5
\end{array}\right] \quad \mathbf{M}=\left[\begin{array}{ccc}
-8 & 6 & 3 \\
-12 & 9 & 4 \\
-6 & 4 & 3
\end{array}\right]
\end{gathered}
$$

(b) A three-dimensional commutative cascade

$$
\begin{gathered}
{\left[\begin{array}{cc}
\frac{2}{7} & \frac{4}{7} \\
\frac{6}{7} & \frac{81}{35}
\end{array}\right] \Longrightarrow \mathbf{J}=\left[\begin{array}{cc}
\frac{91-\sqrt{7441}}{70} & 0 \\
0 & \frac{91+\sqrt{7441}}{70}
\end{array}\right]} \\
{\left[\begin{array}{cc}
\frac{2}{7} & \frac{4}{7} \\
\frac{6}{7} & \frac{81}{35}
\end{array}\right]=\left[\begin{array}{ll}
1 & 0 \\
3 & 1
\end{array}\right]\left[\begin{array}{cc}
\frac{2}{7} & 0 \\
0 & \frac{3}{5}
\end{array}\right]\left[\begin{array}{ll}
1 & 2 \\
0 & 1
\end{array}\right]} \\
\mathbf{L}=\left[\begin{array}{ll}
2 & 0 \\
6 & 3
\end{array}\right] \quad \mathbf{M}=\left[\begin{array}{cc}
7 & -10 \\
0 & 5
\end{array}\right]
\end{gathered}
$$

(c) A commutative cascade does not exist, so a right coprime cascade is computed instead.

Fig. 2. Examples of applying the factoring algorithm to three rational resampling matrices. 